\title{
A regional paediatric rheumatology service
}

\author{
A W CRAFT AND M HALL
}

Department of Child Health and Department of Rheumatology, Royal Victoria Infirmary, Newcastle upon Tyne

SUMMARY A paediatric rheumatology clinic has been established in Newcastle since 1974, and 160 children had been referred to it by the end of 1980 . The proportion of patients in each disease category is similar to that at the national centre at Taplow although far fewer patients with a positive antinuclear antibody test and fewer cases of chronic uveitus are seen. The importance of combining the skills of several disciplines is stressed.

For many years the unit at the Canadian Red Cross Memorial Hospital at Taplow has provided a supraregional service for children with rheumatic disorders and much of the basic work of classifying different disease entities and of developing and evaluating treatment techniques has been done there. The unit at Taplow is soon to close and it has been recommended ${ }^{1}$ that the work should be continued regionally, so some regions are establishing their own units. One such unit in Newcastle upon Tyne was established in 1974 by a rheumatologist and a paediatrician, and we report the work there during its first 6 years. Throughout this paper we use the diagnostic criteria for juvenile chronic arthritis (JCA) and disease categories as defined by the European League Against Rheumatism, ${ }^{2}$ clarified by Ansell in her Heberden oration. ${ }^{3}$ In addition we include patients with juvenile rheumatoid arthritis (JRA) - that is Rose Waaler positive, juvenile onset ankylosing spondylitis, and psoriatic arthritis.

\section{Patients}

Between 1974 and 1980, 160 children aged between
6 months and 16 years were seen at the clinic and 121 of them had JCA (Table). In addition there was a miscellaneous group of 39 children who were found either not to have inflammatory joint disease or to have a disorder that did not fit the diagnostic criteria for JCA. Such disorders included traumatic joint injuries, hypermobile joints, osteochondritis dissecans, and post-rubella arthritis, as well as systemic lupus erythematosus, dermatomyositis, and scleroderma. The main sources of referral were the family doctor $(42 \%)$, paediatricians $(36 \%)$, orthopaedic surgeons $(9 \%)$, and rheumatologists $(7 \%)$. Details of the children with JCA are shown in the Table. Twenty $(17 \%)$ of the children with rheumatic disorders had a close family history of inflammatory arthritis and there were two sets of identical twins both discordant for the disease, one with JCA and the other with psoriatic arthritis. There were two brothers with similar disease. Sixteen $(13 \%)$ children had positive antinuclear antibody tests, $3(19 \%)$ of whom had chronic uveitis. In the entire series, 7 $(6 \%)$ children had chronic uveitis of whom $3(43 \%)$ had positive antinuclear antibody tests. In addition there was one child with ankylosing spondylitis who had acute uveitis. Most children required treatment with non-steroidal anti-inflammatory drugs only, but 30 have also received corticosteroids: 19 penicillamine, 7 gold (sodium aurothiomalate), and 4 chloroquine. Ten children have required orthopaedic surgical intervention for either diagnostic or therapeutic purposes.

Two patients died; both were in the JCA (systemic) group. Each had received treatment with corticosteroids for prolonged periods and died from an encephalitic illness. There were no cases of amyloid.

Table Details of the 160 children

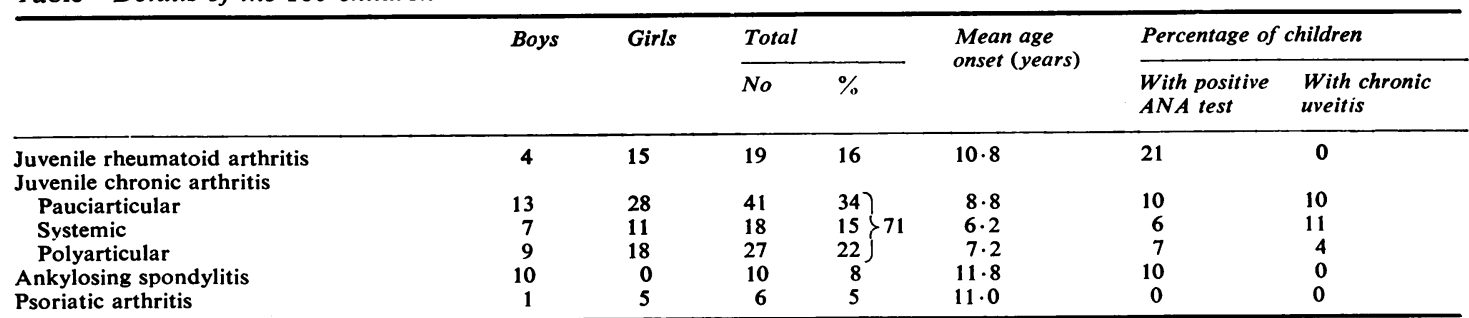

ANA $=$ antinuclear antibody. 
The clinic

A monthly clinic is held in the paediatric outpatient department and the children are seen by a paediatrician and a rheumatologist together with a physiotherapist. An ophthalmologist with a special interest in the rheumatic disorders of children has a clinic on the same afternoon and children are regularly referred. At times outside these monthly clinics children are seen as part of a general paediatric clinic. There is ready access to an orthopaedic surgeon, the dental department, child psychiatrists, and a social worker from the Lady Hoare Trust. The physiotherapist has a particular interest in children and a knowledge of the special techniques of splint-making and physiotherapy necessary for the management of children with JCA. She has visited Taplow and keeps in close contact with her professional colleagues there. Regular sessions are held in a hydrotherapy pool. In order to avoid undue travelling for patients clinics are held once or twice a year in peripheral hospitals where the paediatrician, rheumatologist, and physiotherapist meet their local colleagues. In this way many more children are seen and the knowledge of the special treatment and techniques needed can be imparted to the local physicians and their staff.

\section{Discussion}

Arthritis in children is rare, the prevalence being estimated at 0.65 per 1000 children under age 15 years. ${ }^{4}$ Many cases are mild and self-limiting and can be satisfactorily looked after by most general paediatricians. However, there is a small group of children with chronic unremitting disease who need special management and for whom the ultimate outcome can be greatly improved by early and effective management. Most of the patients seen in the clinic who have been referred by other consultant colleagues are in this category, and many in the past would have been referred to Taplow with consequent expense as well as occasional separation of the child from his family for prolonged periods. A local service reduces the amount of time spent in hospital as most of the treatment can be given on an outpatient basis and only rarely has it been necessary to admit a child and then only for a short period.

The number of children seen in our clinic represents only a portion of the children with these conditions in the region, and the number of children in the different groups does not necessarily correspond with that which would be found in a whole population survey. However, the relative proportions in each subcategory are similar to those reported by Ansell from Taplow. ${ }^{5}$ The low prevalence of positive antinuclear factor and of uveitis is different from other series and we are unable to explain it. The prognosis for JCA has improved considerably in recent years as a result of new methods of medical treatment but at least as important is a greater awareness of the need for early active physiotherapy management. ${ }^{6}$ Far fewer children now end up severely disabled than previously. It is the need for के this expert help early in the disease which makes a $\vec{\circ}$ regional clinic of most value. Any single paediatrician or rheumatologist will see only one or two severe cases each year and it is not feasible to provide appropriate facilities for the care of such a small number of children in every district general o hospital. In addition a regional clinic can act as a $\underset{\infty}{ }$ link with other centres so that new methods of o treatment can be made available for all children.

Patients with JCA may have to be managed for 을 many years and during this time several professional $\rightarrow$ groups are concerned. Primary responsibility is best provided by a paediatrician who has specialised 0 knowledge of the care of children and their families, की and by a rheumatologist with a special expertise in the care of joints. Treatment with the second-line antirheumatic drugs-such as corticosteroids, penicillamine, gold, and chloroquine-can be beneficial but carries a significant morbidity as all such drugs have serious side effects. They should not be withheld for lack of close supervision by a team experienced in their use.

\section{References}

1 Wood P H N. The challenge of arthritis and rheumatism. $A$ report on problems and progress in health care for rheumatic disorders. Based on evidence collected for presentation to the Royal Commission on the National ? Health Service for a Working Party of the British League Against Rheumatism. London: Arthritis and Rheumatism Council, 1977.

2 Munthe E, ed. The care of rheumatic children. Monograph Series, No 3. Basle: EULAR, 1977.

3 Ansell B M. Chronic arthritis in childhood. Ann Rheum 윽 Dis 1977; 37: 107-20.

4 Great Britain Office of Population Censuses and Surveys. $\frac{D}{O}$ Hospital in-patient enquiry; main tables, based on a one in ten sample of NHS patients in hospitals in England and $N$ Wales, 1974. Series MB4, No 2, DHSS, OPCS, and Welsh Office. London: HMSO, 1978.

5 Ansell B M. Rheumatic disorders in childhood. London: Butterworth, 1980: 89.

6 Ansell B M, Wood P H N. Prognosis in juvenile chronic polyarthritis. Clin Rheum Dis 1979; 2: 397-425.

Correspondence to Dr A W Craft, Children's Department, Royal Victoria Infirmary, Queen Victoria Road, Newcastle upon Tyne NE1 4LP.

Received 1 March 1982 